\title{
Klebanov-Strassler black hole
}

\author{
Alex Buchel \\ Department of Applied Mathematics, Department of Physics and Astronomy, \\ University of Western Ontario, \\ London, Ontario N6A 5B\%, Canada \\ Perimeter Institute for Theoretical Physics, \\ Waterloo, Ontario N2J 2W9, Canada \\ E-mail: abuchel@uwo.ca
}

ABSTRACT: We construct a black hole solution on warped deformed conifold in type IIB supergravity with fluxes. The black hole has translationary invariant horizon and is a holographic dual to a thermal homogeneous and isotropic state of a cascading $\mathrm{SU}(K+P) \times \mathrm{SU}(K) \mathcal{N}=1$ supersymmetric gauge theory with spontaneously broken chiral symmetry. We discuss thermal properties of the new black hole solutions. We comment on implications of the new black hole solutions for the landscape of KKLT de Sitter vacua in string theory.

KEYWORDS: Black Holes in String Theory, Gauge-gravity correspondence, Holography and quark-gluon plasmas, Flux compactifications

ARXIV EPRINT: 1809.08484 


\section{Contents}

1 Introduction 1

2 Holography: phases of the cascading gauge theory 4

2.1 Canonical ensemble 6

$\begin{array}{lll}2.2 & \text { Microcanonical ensemble } & 7\end{array}$

3 Landscape: KKLT de Sitter vacua in string theory 4

4 Conclusion $\quad 9$

A Technical details on construction of Klebanov-Strassler black hole $\quad 10$

$\begin{array}{lll}\text { A.1 Effective action and boundary asymptotics } & 10\end{array}$

A.2 Holographic renormalization, thermodynamic quantities and horizon D3

$\begin{array}{ll}\text { Maxwell charge } & 13\end{array}$

$\begin{array}{lll}\text { A.3 Numerical procedure } & 14\end{array}$

\section{Introduction}

A conifold is a complex 3 -dimensional manifold described by the following equation in $\mathbb{C}^{4}$,

$$
\sum_{n=1}^{4} z_{n}^{2}=0 .
$$

Owing to explicitly known Ricci-flat metric [1], the conifold appeared prominently in string theory and supergravity:

- In a holographic context [2], placing a large number $K$ of $D 3$ branes at the tip of the (singular) conifold realizes a duality for $\mathcal{N}=1 \mathrm{SU}(K) \times \mathrm{SU}(K)$ superconformal gauge theory, also known as Klebanov-Witten gauge theory [3]. Further wrapping $P$ $D 5$ branes on a 2-cycle of a conifold realizes a correspondence with $\mathcal{N}=1 \mathrm{SU}(K+$ $P) \times \mathrm{SU}(K)$ Klebanov-Strassler $(\mathrm{KS})$ gauge theory [4]. KS gauge theory undergoes an infinite sequence - a cascade - of self-similar Seiberg duality [5] transformations in the UV; it confines with the spontaneous chiral symmetry breaking in the IR.

- In [6] (GKP) it was pointed out that type IIB string theory compactified on warped throat geometries with fluxes (local version of which is precisely that of KS gauge theory gravitational dual) produces no-scale $\mathcal{N}=1$ supersymmetric Minkowski vacua, naturally generating large hierarchies of physical scales. GKP compactifications fix all complex structure moduli, leaving at least a single Kähler modulus (an overall volume of a compact 6-dimensional manifold) unfixed. KKLT [7] further argued that 
non-perturbative corrections in GKP compactifications fix the overall volume Kähler modulus leading to $\mathcal{N}=1$ SUSY preserving $A d S_{4}$ vacua. Adding $\overline{D 3}$ to compactifications lifts AdS vacua to de Sitter. KKLT construction has been taken as the primary evidence for a landscape of de Sitter vacua in string theory.

In this paper we present new results regarding black hole solutions in type IIB supergravity on warped deformed conifold with fluxes. Our results are relevant both for holography and the landscape:

- Black hole geometries in holography represent gravitational dual to thermal states of the boundary gauge theories [8]. Although the chiral symmetry is spontaneously broken in the vacuum of KS gauge theory, it is expected to be restored at sufficiently high temperature. This predicts the existence of black hole solutions dual to thermal states of the cascading gauge theory plasma with unbroken chiral symmetry [9]. Such black holes resolve the singularity of the Klebanov-Tseytlin [10] geometry [9, 11, 12]. KS gauge theory confines in the infrared - the holographic dual of this (firstorder) phase transition was established in $[13]^{1}(\mathrm{ABK})$. ABK black hole solutions represent the gravitational dual to deconfined thermal states of KS gauge theory with unbroken chiral symmetry. These black holes cease to exist below some critical temperature $T<T_{u}$ [15], where they join a perturbatively unstable branch (with a negative specific heat and condensation of the hydrodynamic sound modes). ABK black holes are also perturbatively unstable for $T<T_{\chi \mathrm{SB}}=1.00869(0) T_{u}$ towards development of the chiral symmetry breaking $(\chi \mathrm{SB})$ condensates in KS gauge theory plasma [16]. The end point of the $\chi \mathrm{SB}$ breaking instability in ABK black holes would produce Klebanov-Strassler black hole - gravitational backgrounds dual to homogeneous and isotropic thermal deconfined states of KS gauge theory plasma with spontaneously broken chiral symmetry. Thermodynamics of KS black holes will be discussed in section 2 .

- The most controversial aspect of the KKLT construction of de Sitter vacua is the uplift of $A d S_{4}$ non-perturbative GKP vacua due to $\overline{D 3}$ branes. The backreacted solutions corresponding to smeared $\overline{D 3}$ branes at the tip of the KS solution were argued to be singular [17-20]. Because the singularity is localized, it must be possible to study it in the local geometry of the GKP background - the noncompact gravitational dual to KS gauge theory. The following strategy was proposed in [21]: if the singularity due to $\overline{D 3}$ branes at the conifold is physical, it should be possible to shield it with a horizon [22]: i.e. there must exist a black hole solution on the conifold that carries a negative D3 brane charge at the horizon. Black holes with negative D3 brane horizon charge have not been found for a conifold with an unbroken U(1) symmetry (a gravitational dual to a chiral symmetry in KS gauge theory); neither were found black holes with negative charge where this U(1) symmetry is broken explicitly [21]. KKLT construction requires warped deformed conifolds with spontaneous symmetry

\footnotetext{
${ }^{1}$ To understand the thermodynamics of chirally symmetric states of the cascading gauge theory plasma it was important to understand the holographic renormalization of the theory [14].
} 
breaking $\mathrm{U}(1) \supset \mathbb{Z}_{2}$ — thus, one needs to search for negative $D 3$ brane charge KS black holes. We report on this in section 3 .

Constructions of Klebanov-Strassler black holes have been attempted in the past: the complete ansatz for the metric and the background fluxes was proposed in [16]. ${ }^{2}$ The latter reference contains the consistent set of equations of motion, which incorporates as special cases all previously known solutions [11-13], dual to thermal states of cascading gauge theory with unbroken chiral symmetry (the conifold deformation parameter is switched off). As we already mentioned, the background ansatz of [16] identifies (linearized) perturbative instability of Klebanov-Tseytlin (ABK) black holes at $T<T_{\chi \mathrm{SB}}$ due to fluctuations associated with the conifold deformation parameter - in the cascading gauge theory language this is a coupled set of two dimension- 3 operators $\mathcal{O}_{3}^{1,2}$ and a dimension-7 operator $\mathcal{O}_{7}$, see section 3 of [16]. Klebanov-Strassler black hole is a solution within the metric ansatz [16] with non-linear thermal expectation values $\mathcal{O}_{3}^{1,2}$ and $\mathcal{O}_{7}$. It is important to distinguish black hole solutions where the non-normalizable components of gravitational background fields dual to $\mathcal{O}_{3}^{1,2}$ operators vanish or are nonzero: the genuine Klebanov-Strassler black hole, dual to thermal deconfined phase of cascading gauge theory with spontaneously broken chiral symmetry is the former; black hole solutions with nonzero non-normalizable components of the fields dual to $\mathcal{O}_{3}^{1,2}$ operators describe explicit breaking of chiral symmetry by gaugino mass terms. Thermal states of cascading gauge theory with explicit breaking of chiral symmetry due to gaugino mass terms were extensively studied in section 4 of [16]. ${ }^{3}$ It was demonstrated in [16] that thermal states in mass-deformed cascading theory at $T<T_{\chi \mathrm{SB}}$ reduce to chirally-symmetric Klebanov-Tseytlin black holes in the limit of vanishing gaugino masses, see figure 4 in [16] - this was taken as an evidence that Klebanov-Strassler black holes do not exist. Both a conceptual and a technical advance allowed a successful construction of Klebanov-Strassler black hole reported in this paper, 18 years since the first work on a subject [9]:

- At a conceptual level, it was realized that KS black holes could never dominated the canonical ensemble, and thus might not simply exist for $T<T_{\chi \mathrm{SB}}$, in agreement with [16]. For this to be true, the thermal phase diagram of the system should resemble the one of the "Exotic Hairy Black Holes" first discovered in [26]. As we will see in section 2.1 below, this is indeed the case.

- The technical difficulty in numerically constructing KS black holes is the nonzero value of the thermal expectation value of $\mathcal{O}_{7}$ operator. This implies that in numerical solutions one must keep computational control over the boundary asymptotics (the radial coordinate $r \rightarrow \infty$ ) of the fields to level $\mathcal{O}\left(r^{-7}\right)$, while the leading asymptotic of the fields is $\sim \ln r$. Furthermore, this thermal expectation value vanishes as $\mathcal{O}_{7} \sim$ $\left|T-T_{\chi \mathrm{SB}}\right|^{1 / 2} \rightarrow 0$ at the temperature of the spontaneous chiral symmetry breaking $T_{\chi \mathrm{SB}}$. (It is precisely to circumvent this difficulty, it was proposed in [16] to break

\footnotetext{
${ }^{2}$ In later work, [25], the authors did not identify all the required thermal condensates in cascading gauge theory plasma.

${ }^{3}$ These computations were independently reproduced in [21].
} 
chiral symmetry explicitly, and then construct KS black holes in the limit of vanishing gaugino masses.) In this paper we solved the technical problem, without introducing gaugino mass terms, by developing new computational Mathematica scripts to solve relevant differential equations with arbitrary numerical precision.

In the next two sections we present results of relevance to cascading gauge theory holography and to the landscape of KKLT de Sitter vacua, omitting all the technical details. All the necessary technical details are reviewed (following [16]) in appendix.

\section{Holography: phases of the cascading gauge theory}

In our review of the cascading gauge theory and the thermodynamics of its chirally symmetric states we closely follow [16].

Klebanov-Strassler gauge theory is $\mathcal{N}=1$ four-dimensional supersymmetric $\mathrm{SU}(K+$ $P) \times \mathrm{SU}(K)$ gauge theory with two chiral superfields $A_{1}, A_{2}$ in the $(K+P, \bar{K})$ representation, and two fields $B_{1}, B_{2}$ in the $(\overline{K+P}, K)$. This gauge theory has two gauge couplings $g_{1}, g_{2}$ associated with two gauge group factors, and a quartic superpotential

$$
W \sim \operatorname{Tr}\left(A_{i} B_{j} A_{k} B_{\ell}\right) \epsilon^{i k} \epsilon^{j \ell} .
$$

When $P=0$ above theory flows in the infrared to a superconformal fixed point, commonly referred to as Klebanov-Witten theory. At the IR fixed point KW gauge theory is strongly coupled - the superconformal symmetry together with $\mathrm{SU}(2) \times \mathrm{SU}(2) \times \mathrm{U}(1)$ global symmetry of the theory implies that anomalous dimensions of chiral superfields $\gamma\left(A_{i}\right)=\gamma\left(B_{i}\right)=-\frac{1}{4}$, i.e. non-perturbatively large.

When $P \neq 0$, conformal invariance of the above $\mathrm{SU}(K+P) \times \mathrm{SU}(K)$ gauge theory is broken. It is useful to consider an effective description of this theory at energy scale $\mu$ with perturbative couplings $g_{i}(\mu) \ll 1$. It is straightforward to evaluate NSVZ beta-functions for the gauge couplings. One finds that while the sum of the gauge couplings does not run

$$
\frac{d}{d \ln \mu}\left(\frac{\pi}{g_{s}} \equiv \frac{4 \pi}{g_{1}^{2}(\mu)}+\frac{4 \pi}{g_{2}^{2}(\mu)}\right)=0,
$$

the difference between the two couplings is

$$
\frac{4 \pi}{g_{2}^{2}(\mu)}-\frac{4 \pi}{g_{1}^{2}(\mu)} \sim P\left[3+2\left(1-\gamma_{i j}\right)\right] \ln \frac{\mu}{\Lambda},
$$

where $\Lambda$ is the strong coupling scale of the theory and $\gamma_{i j}$ is an anomalous dimension of operators $\operatorname{Tr} A_{i} B_{j}$. Given (2.3) and (2.2) it is clear that the effective weakly coupled description of $\mathrm{SU}(K+P) \times \mathrm{SU}(K)$ gauge theory can be valid only in a finite-width energy band centered about $\mu$ scale: extending effective description both to the UV and to the IR one necessarily encounters strong coupling in one or the other gauge group factor. To extend the theory past the strongly coupled region(s) one must perform a Seiberg duality. In KS gauge theory a Seiberg duality transformation is a self-similarity transformation of the effective description so that $K \rightarrow K-P$ as one flows to the IR, or $K \rightarrow K+P$ as 
the energy increases. Thus, extension of the effective $\mathrm{SU}(K+P) \times \mathrm{SU}(K)$ description to all energy scales involves a cascade of Seiberg dualities where the rank of the gauge group changes with energy according to

$$
K=K(\mu) \sim 2 P^{2} \ln \frac{\mu}{\Lambda}
$$

at least as $\mu \gg \Lambda$. Although there are infinitely many duality cascade steps in the UV, there is only a finite number of duality transformations as one flows to the IR (from a given scale $\mu$ ). The space of vacua of a generic cascading gauge theory was studied in details in [23]. When $K(\mu)$ is an integer multiple of $P$, the cascading gauge theory confines in the infrared with a spontaneous breaking of the chiral symmetry.

The thermal phase digram of homogeneous and isotropic states in cascading gauge theory plasma represents competition between three phases:

- (A): confined phase with spontaneously broken chiral symmetry;

- (B): deconfined chirally symmetric phase;

- (C): deconfined phase with spontaneously broken chiral symmetry.

Correspondingly, in a dual gravitational description we have:

- (Ah): thermal KS geometry, i.e. Klebanov-Strassler vacuum solution with periodically identified Euclidean time direction,

$$
t_{E} \sim t_{E}+\frac{1}{T}
$$

In this phase all the thermodynamic potentials vanish: the free energy density $\mathcal{F}$, the energy density $\mathcal{E}$ and the entropy density $s$. There are nonvanishing condensates of two dimension-3 operators (dual to chiral symmetry breaking gaugino condensates of both gauge group factors), and a condensate of a dimension-6 operator [16].

- (Bh): Klebanov-Tseytlin black hole. In this phase we have nonvanishing $\{\mathcal{F}, \mathcal{E}, s\}$. There are nonvanishing condensates of two dimension- 4 operators, a dimension- 6 operator and a dimension- 8 operator [13]. Condensates of the chiral symmetry breaking operators vanish.

- (Ch): Klebanov-Strassler black hole. In this phase we have nonvanishing $\{\mathcal{F}, \mathcal{E}, s\}$. In additional to the condensates present in $(\mathrm{Bh})$, we have condensates of a pair of chiral symmetry breaking dimension-3 operators (as in (Ah)) and an additional condensate of a dimension-7 operator (also breaking the chiral symmetry) [16].

Phase transition between $A \leftrightarrow B$ is of the first-order [13].

We now turn to a detailed discussion of the phase diagram of the cascading gauge theory plasma. At temperatures $T \gg \Lambda$ the cascading plasma is in the deconfined phase with an 


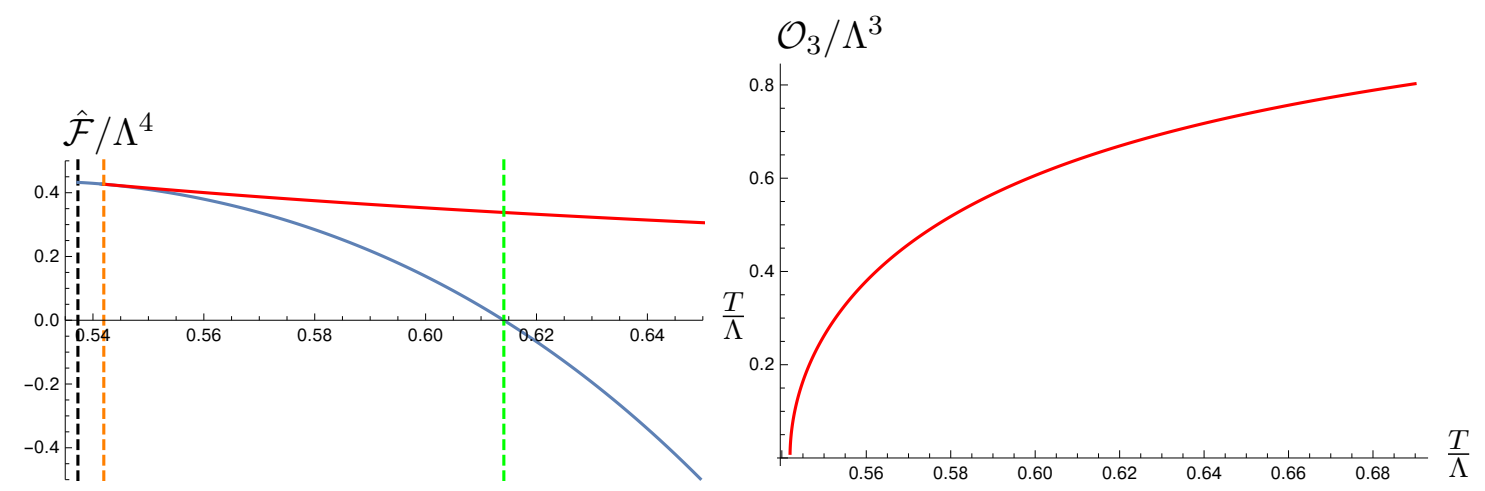

Figure 1. Phase diagram of the cascading gauge theory plasma in canonical ensemble. Solid blue line represents deconfined, chirally symmetric phase of the theory — the gravitational dual to these thermal states is a Klebanov-Tseytlin black hole. Solid red lines represent deconfined phase of the theory with the spontaneously broken chiral symmetry - the gravitational dual to these thermal states is a Klebanov-Strassler black hole. Left panel shows the free energy density versus the temperature of the symmetric and the symmetry broken phases. The vertical dashed green line indicates the first-order confinement-deconfinement phase transition; the vertical dashed orange line indicates the onset of the perturbative instability of the chirally symmetric phase; the vertical dashed black line indicates the onset of the instability of sound waves in chirally symmetric phase of the cascading plasma. Right panel shows a critical behavior of one of the dimension- 3 condensates in the chiral symmetry broken phase of the cascading plasma.

unbroken chiral symmetry (B) $[9,11,12]$. Here, the temperature-dependent effective rank $K(T)$ of the cascading theory is large, compare to $P[14]$ :

$$
\frac{K(T)}{P^{2}}=\frac{1}{2} \ln \left(\frac{64 \pi^{4}}{81} \times \frac{s T}{\Lambda^{4}}\right) \quad \Longrightarrow \quad \frac{K(T)}{P^{2}} \approx 2 \ln \frac{T}{\Lambda}, \quad T \gg \Lambda .
$$

To leading order at higher temperature, the pressure $\mathcal{P}=-\mathcal{F}$ and the energy density $\mathcal{E}$ are given by [14]

$$
\begin{aligned}
\frac{\mathcal{P}}{s T} & =\frac{1}{4}\left(1-\frac{P^{2}}{K(T)^{2}}+\mathcal{O}\left(\frac{P^{4}}{K(T)^{2}}\right)\right) \\
\frac{\mathcal{E}}{s T} & =\frac{3}{4}\left(1+\frac{1}{3} \frac{P^{2}}{K(T)^{2}}+\mathcal{O}\left(\frac{P^{4}}{K(T)^{2}}\right)\right) .
\end{aligned}
$$

At low temperature/energy density we need to distinguish canonical (see figure 1) and microcanonical (see figure 2) ensembles.

\subsection{Canonical ensemble}

The deconfined chirally symmetry phase (B) (represented by a solid blue curve) extends to temperature [13]

$$
T_{c}=0.6141111(3) \Lambda
$$

below which its free energy density

$$
\hat{\mathcal{F}}=\frac{128 \pi^{4}}{81 P^{4}} \mathcal{F}
$$


becomes positive. This signifies the first-order phase transition to the confined phase with the spontaneously broken chiral symmetry (A). This phase transition is denoted by a vertical dashed green line. Since $A \leftrightarrow B$ phase transition proceeds via bubble nucleation, it is non-perturbative. At temperature

$$
T_{\chi \mathrm{SB}}=0.54195(5) \Lambda
$$

the meta-stable phase (B) becomes perturbatively unstable due to chiral symmetry breaking fluctuations [16]. This instability is denoted by a vertical dashed orange line. At temperature

$$
T_{u}=0.537286 \Lambda,
$$

the phase (B) terminates joining a perturbatively unstable branch of the theory with a negative specific heat [15]. The branch with a negative specific heat has dynamical instability leading to a breakdown of spatial homogeneity in plasma: the sound waves are unstable [24]. The terminal temperature $T_{u}$ is denoted by a vertical dashed black line. Note the hierarchy of critical temperatures of homogeneous and isotropic thermal states in cascading plasma:

$$
T_{u}<T_{\chi \mathrm{SB}}<T_{c} .
$$

A natural expectation is that the deconfined phase with spontaneously broken chiral symmetry - the phase (C) - should bifurcate from (B) at $T=T_{\chi \mathrm{SB}}$ where the fluctuations associated with this symmetry breaking become unstable. The end point of the instability for $T<T_{\chi \mathrm{SB}}$ would be Klebanov-Strassler black hole. Until this work, the searches for the KSBH were unsuccessful. The right panel of figure 1 provides a reason: ${ }^{4}$ although the $\chi \mathrm{SB}$ fluctuations are unstable for $T \leq T_{\chi \mathrm{SB}}$, the $\mathrm{KSBH}$ exists only for $T \geq T_{\chi \mathrm{SB}}$. In other words, the KSBH is in a class of exotic black holes originally identified in [26]. ${ }^{5}$ The phase $(\mathrm{C})$ of the cascading gauge theory plasma is denoted by a solid red curve: it has a higher free energy density than the phase (B) at the corresponding temperature and thus never dominates in macrocanonical ensemble.

\subsection{Microcanonical ensemble}

Microcanonical ensemble is relevant for dynamical questions (thermalization and equilibration) of gauge theory plasma. Figure 2 presents the phase diagram of the cascading gauge theory in microcanonical ensemble. The solid blue curve denotes phase (B), and the solid red curve denotes phase (C). Similar to (2.9) we introduced

$$
\hat{\mathcal{E}}=\frac{128 \pi^{4}}{81 P^{4}} \mathcal{E}, \quad \hat{s}=\frac{128 \pi^{4}}{81 P^{4}} s .
$$

A vertical orange line

$$
\hat{\mathcal{E}}_{\chi \mathrm{SB}}=1.270093(1) \Lambda^{4}
$$

indicates the onset of the chiral symmetry breaking instability [16]. Notice that here the phase (C) exists for $\mathcal{E} \leq \mathcal{E}_{\chi \mathrm{SB}}$ and dominates over the phase (B). In other words, insisting

\footnotetext{
${ }^{4}$ Over the years, the author was searching for the KSBH at $T<T_{\chi \mathrm{SB}}$.

${ }^{5}$ See also [27].
} 

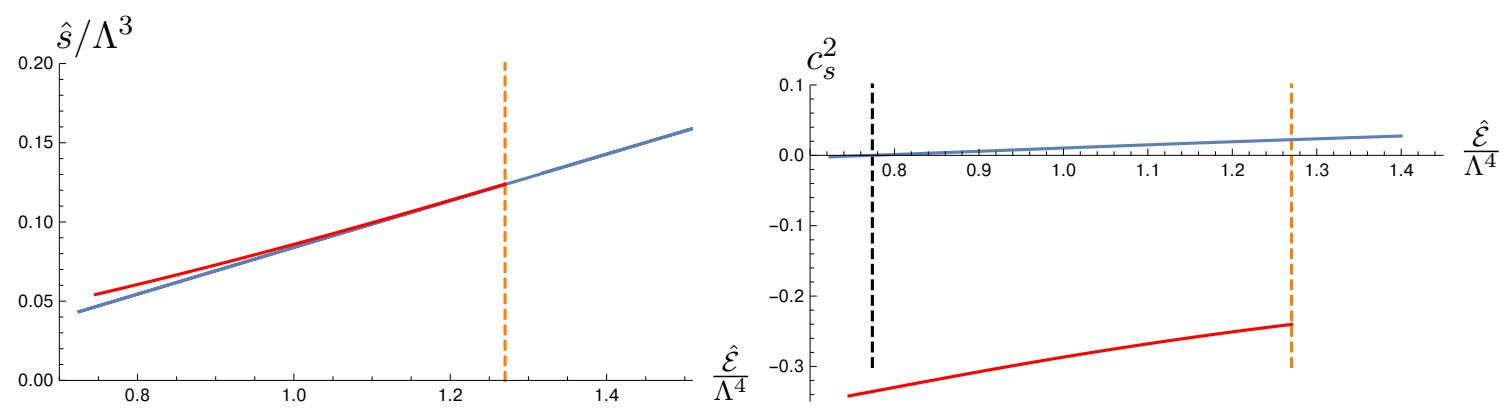

Figure 2. Phase diagram of the cascading gauge theory plasma in microcanonical ensemble. Solid blue lines represent deconfined, chirally symmetric phase of the theory - the gravitational dual to these thermal states is a Klebanov-Tseytlin black hole. Solid red lines represent deconfined phase of the theory with the spontaneously broken chiral symmetry - the gravitational dual to these thermal states is a Klebanov-Strassler black hole. Left panel shows the entropy density versus the energy density of the symmetric and the symmetry broken phases. The vertical dashed orange line indicates the onset of the perturbative instability of the chirally symmetric phase; the vertical dashed black line indicates the onset of the instability of the sound waves in chirally symmetric phase of the cascading plasma. Right panel shows the speed of sound waves squared in symmetric and symmetry broken phases of the cascading plasma.

on homogeneous and isotropic evolution, the KSBH is the end point of the perturbative instability of the KTBH at sufficiently low energy densities. Such a phenomenon in a context of exotic black holes was identified in [28].

The KSBH is both thermodynamically and dynamically unstable. The right panel of figure 2 presents the speed of sound waves as a function of the energy density for (B) (blue curve) and (C) (red curve) phases of the cascading gauge theory plasma. A vertical black line

$$
\hat{\mathcal{E}}_{u}=0.723488 \Lambda^{4}
$$

indicates the onset of the perturbative instability in phase (B) associates with the breaking of the translational invariance due to the condensation of the hydrodynamic sound waves [15].

\section{Landscape: KKLT de Sitter vacua in string theory}

Following [21], we compute the Maxwell D3-brane charge of the conifold black hole horizons $Q_{b}^{\text {D3 }}$. A negative value of the horizon charge would indicate that the anti-D3 brane singularity is physical, according to classification [22]. The results of the computations are presented in figure 3. The solid blue curve represents the Maxwell charge of KTBH, and the solid red curve represents the charge of the KSBH. Both charges are never negative; for $\mathcal{E}<\mathcal{E}_{\chi \mathrm{SB}}$, when the $\mathrm{KSBH}$ has a higher entropy then the corresponding energy density KTBH,

$$
\left.Q_{b}^{\mathrm{D} 3}\right|_{\mathrm{KSBH}}>\left.Q_{b}^{\mathrm{D} 3}\right|_{\mathrm{KTBH}}
$$




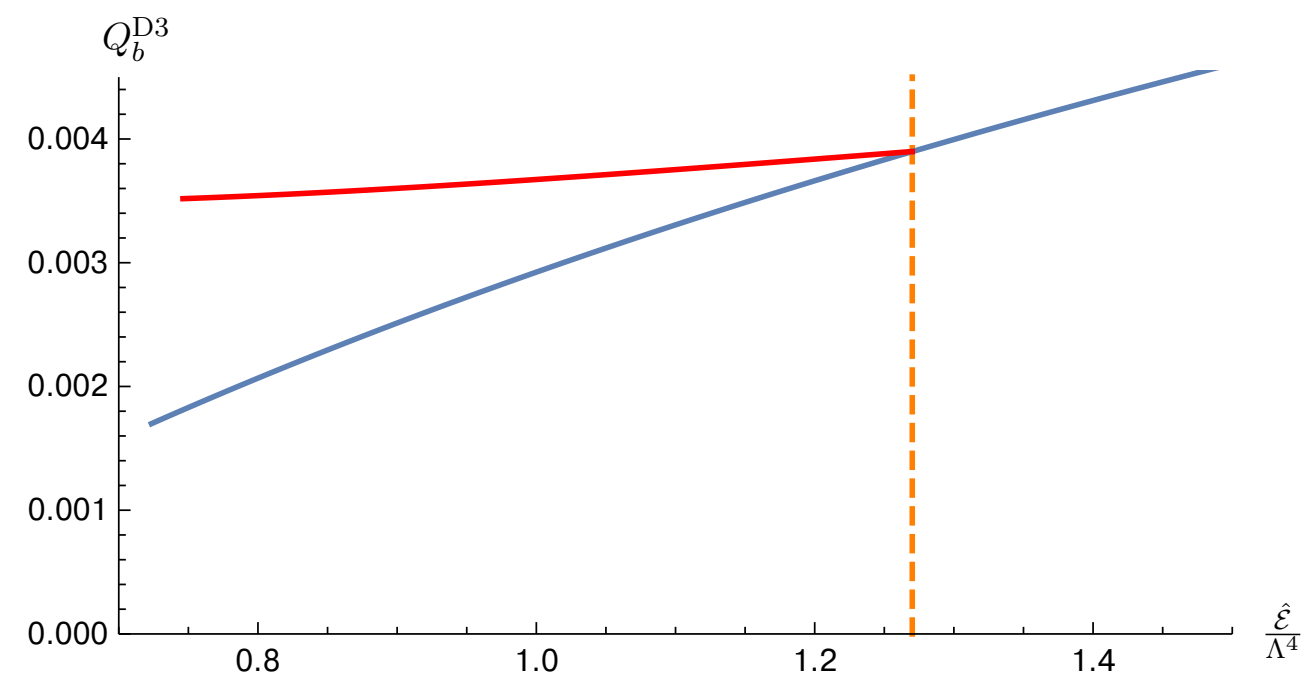

Figure 3. The Maxwell D3-brane charge of Klebanov-Tseytlin black holes (solid blue line) and Klebanov-Strassler black holes (solid red line). Dashed orange line indicates onset of the perturbative instability of KT black holes, spontaneously breaking $U(1) \supset \mathbb{Z}_{2}$ chiral symmetry. The end point of the evolution of the instability of a KT black hole for $\mathcal{E}<\mathcal{E}_{\chi \mathrm{SB}}$, under homogeneity and isotropy constraints of the horizon, is a KS black hole at the corresponding energy density.

\section{Conclusion}

In this paper we reported on the physical properties of Klebanov-Strassler black holes on warped deformed conifold with fluxes. These black holes are important for understanding of the holographic correspondence for the confining KS gauge theory [4]. They were also longsought objects in the context of KKLT de Sitter vacua constructions in string theory [7].

We established the existence of KSBHs. We determined that these black holes have a negative specific heat, and are dynamically unstable due to the hydrodynamic sound wave fluctuations (breaking the homogeneity of the horizon).

KSBHs realize the homogeneous and isotropic deconfined phase of the cascading gauge theory plasma with spontaneously broken chiral symmetry. While the corresponding phase never dominates in canonical ensemble, it has a higher entropy density compare to the homogeneous and isotropic phase with unbroken chiral symmetry at the corresponding energy density (the gravitational dual to KTBHs) below some critical energy density, i.e. $\mathcal{E}<\mathcal{E}_{\chi \mathrm{SB}}$. Of course, since ultimately the homogeneity assumption is not valid (KSBHs are dynamically unstable), the equilibrium states of the cascading gauge theory below $\mathcal{E}_{\chi \mathrm{SB}}$ remain unknown - for sure they can not be homogeneous and isotropic.

Finally, we demonstrated that KSBHs can not shield (conjectured) anti-D3 brane singularity on the warped deformed conifold with fluxes.

\section{Acknowledgments}

Research at Perimeter Institute is supported by the Government of Canada through Industry Canada and by the Province of Ontario through the Ministry of Research \& Innovation. This work was further supported by NSERC through the Discovery Grants program. 


\section{A Technical details on construction of Klebanov-Strassler black hole}

Following [16], we present here technical details necessary to reproduce the results reported in this paper.

\section{A.1 Effective action and boundary asymptotics}

Five-dimensional effective action describing black holes on warped deformed conifold with fluxes and $\mathrm{SU}(2) \times \mathrm{SU}(2) \times \mathbb{Z}_{2}$ global symmetry takes form:

$$
\begin{aligned}
S_{5}= & \frac{108}{16 \pi G_{5}} \int_{\mathcal{M}_{5}} \operatorname{vol}_{\mathcal{M}_{5}} \Omega_{1} \Omega_{2}^{2} \Omega_{3}^{2}\left\{R_{10}-\frac{1}{2}(\nabla \Phi)^{2}\right. \\
& -\frac{1}{2} e^{-\Phi}\left(\frac{\left(h_{1}-h_{3}\right)^{2}}{2 \Omega_{1}^{2} \Omega_{2}^{2} \Omega_{3}^{2}}+\frac{1}{\Omega_{3}^{4}}\left(\nabla h_{1}\right)^{2}+\frac{1}{\Omega_{2}^{4}}\left(\nabla h_{3}\right)^{2}\right) \\
& -\frac{1}{2} e^{\Phi}\left(\frac{2}{\Omega_{2}^{2} \Omega_{3}^{2}}\left(\nabla h_{2}\right)^{2}+\frac{1}{\Omega_{1}^{2} \Omega_{2}^{4}}\left(h_{2}-\frac{P}{9}\right)^{2}+\frac{1}{\Omega_{1}^{2} \Omega_{3}^{4}} h_{2}^{2}\right) \\
& \left.-\frac{1}{2 \Omega_{1}^{2} \Omega_{2}^{4} \Omega_{3}^{4}}\left(4 \Omega_{0}+h_{2}\left(h_{3}-h_{1}\right)+\frac{1}{9} P h_{1}\right)^{2}\right\},
\end{aligned}
$$

where $R_{10}$ is given by

$$
\begin{aligned}
R_{10}=R_{5} & +\left(\frac{1}{2 \Omega_{1}^{2}}+\frac{2}{\Omega_{2}^{2}}+\frac{2}{\Omega_{3}^{2}}-\frac{\Omega_{2}^{2}}{4 \Omega_{1}^{2} \Omega_{3}^{2}}-\frac{\Omega_{3}^{2}}{4 \Omega_{1}^{2} \Omega_{2}^{2}}-\frac{\Omega_{1}^{2}}{\Omega_{2}^{2} \Omega_{3}^{2}}\right)-2 \square \ln \left(\Omega_{1} \Omega_{2}^{2} \Omega_{3}^{2}\right) \\
& -\left\{\left(\nabla \ln \Omega_{1}\right)^{2}+2\left(\nabla \ln \Omega_{2}\right)^{2}+2\left(\nabla \ln \Omega_{3}\right)^{2}+\left(\nabla \ln \left(\Omega_{1} \Omega_{2}^{2} \Omega_{3}^{2}\right)\right)^{2}\right\},
\end{aligned}
$$

where $R_{5}$ is the five dimensional Ricci scalar of the metric

$$
d s_{5}^{2}=g_{\mu \nu}(y) d y^{\mu} d y^{\nu}, \quad y^{\mu}=\left\{t, x_{1}, x_{2}, x_{3}\right\} .
$$

The full ten-dimensional metric ansatz is

$$
d s_{10}^{2}=g_{\mu \nu}(y) d y^{\mu} d y^{\nu}+\Omega_{1}^{2}(y) g_{5}^{2}+\Omega_{2}^{2}(y)\left[g_{3}^{2}+g_{4}^{2}\right]+\Omega_{3}^{2}(y)\left[g_{1}^{2}+g_{2}^{2}\right],
$$

where

$$
\begin{aligned}
g_{1} & =\frac{\alpha^{1}-\alpha^{3}}{\sqrt{2}}, \quad g_{2}=\frac{\alpha^{2}-\alpha^{4}}{\sqrt{2}}, \\
g_{3} & =\frac{\alpha^{1}+\alpha^{3}}{\sqrt{2}}, \quad g_{4}=\frac{\alpha^{2}+\alpha^{4}}{\sqrt{2}}, \\
g_{5} & =\alpha^{5}, \\
\alpha^{1} & =-\sin \theta_{1} d \phi_{1}, \quad \alpha^{2}=d \theta_{1}, \\
\alpha^{3} & =\cos \psi \sin \theta_{2} d \phi_{2}-\sin \psi d \theta_{2}, \\
\alpha^{4} & =\sin \psi \sin \theta_{2} d \phi_{2}+\cos \psi d \theta_{2}, \\
\alpha^{5} & =d \psi+\cos \theta_{1} d \phi_{1}+\cos \theta_{2} d \phi_{2} .
\end{aligned}
$$


We find it convenient to introduce

$$
\begin{aligned}
& h_{1}=\frac{1}{P}\left(\frac{K_{1}}{12}-36 \Omega_{0}\right), \quad h_{2}=\frac{P}{18} K_{2}, \quad h_{3}=\frac{1}{P}\left(\frac{K_{3}}{12}-36 \Omega_{0}\right), \\
& \Omega_{1}=\frac{1}{3} f_{c}^{1 / 2} h^{1 / 4}, \quad \Omega_{2}=\frac{1}{\sqrt{6}} f_{a}^{1 / 2} h^{1 / 4}, \quad \Omega_{3}=\frac{1}{\sqrt{6}} f_{b}^{1 / 2} h^{1 / 4} .
\end{aligned}
$$

The five-dimensional metric ansatz for the KSBH is

$$
d s_{5}^{2}=H^{-1 / 2}\left(-(1-x)^{2} d t^{2}+d x_{1}^{2}+d x_{2}^{2}+d x_{3}^{2}\right)+g_{x x} d x^{2}
$$

where

$$
H(x)=\left(2 x-x^{2}\right) h(x)
$$

$x$ is the compactified radial coordinate:

$$
x \in(0,1)
$$

From the effective action (A.1) we obtained non-linear system of ODEs for 8 functions (see [16] for the explicit form of the system):

- the three flux functions: $\left\{K_{1}, K_{2}, K_{3}\right\}$;

- the overall warp factor of deformed $T^{1,1}:\{h\}$;

- the three deformation warp factors "inside" $T^{1,1}:\left\{f_{a}, f_{b}, f_{c}\right\}$;

- the string coupling constant: $\{g\}$

All equations of motion are second order in the radial derivative $\frac{d}{d x}$ (the second derivatives enter linearly the system), thus we need $8 \times 2=16$ integration constants to specify a numerical solution.

The UV asymptotic corresponds to $x \rightarrow 0_{+}$. We find:

$$
\begin{aligned}
K_{1} & =P^{2} g_{0} k_{s}-\frac{1}{2} P^{2} g_{0} \ln x+\sum_{n=3}^{\infty} \sum_{k} k_{1 n k} x^{n / 4} \ln ^{k} x, \\
K_{2} & =1+\sum_{n=3}^{\infty} \sum_{k} k_{2 n k} x^{n / 4} \ln ^{k} x, \\
K_{3} & =P^{2} g_{0} k_{s}-\frac{1}{2} P^{2} g_{0} \ln x+\sum_{n=3}^{\infty} \sum_{k} k_{3 n k} x^{n / 4} \ln ^{k} x, \\
f_{a} & =a_{0}\left(1+\sum_{n=3}^{\infty} \sum_{k} f_{\text {ank }} x^{n / 4} \ln ^{k} x\right), \\
f_{b} & =a_{0}\left(1+\sum_{n=3}^{\infty} \sum_{k} f_{b n k} x^{n / 4} \ln ^{k} x\right), \\
f_{c} & =a_{0}\left(1+\sum_{n=2}^{\infty} \sum_{k} f_{c n k} x^{n / 4} \ln ^{k} x\right),
\end{aligned}
$$




$$
\begin{aligned}
h & =\frac{P^{2} g_{0}}{a_{0}^{2}}\left(\frac{1}{8}+\frac{k_{s}}{4}\right)-\frac{P^{2} g_{0}}{8 a_{0}^{2}} \ln x+\sum_{n=2}^{\infty} \sum_{k} h_{n k} x^{n / 4} \ln ^{k} x, \\
g & =g_{0}\left[1+\sum_{n=2}^{\infty} \sum_{k} g_{n k} x^{n / 2} \ln ^{k} x\right] .
\end{aligned}
$$

The expansion depends on 4 microscopic parameters

$$
\left\{P^{2}, g_{0}, a_{0}, k_{s}\right\},
$$

- $P^{2} g_{0}$ - the dimensionless parameter of the cascading theory (which must be large for the gravity approximation to be valid);

- $a_{0}^{2}=4 \pi G_{5} s T$ - where $s$ is the entropy density and $T$ is the temperature of KSBH;

- the strong coupling scale $\Lambda$ of the cascading gauge theory is given by

$$
k_{s} \equiv \frac{1}{2} \ln \left(\frac{a_{0}^{2}}{\Lambda^{4}}\right)=\frac{1}{2} \ln \left(\frac{4 \pi G_{5} s T}{\Lambda^{4}}\right) .
$$

Besides (A.19), the expansions (A.11)-(A.18) are characterized by 7 expectation values:

those of dimension-3 operators:

$$
\left\{d f_{0} \equiv \frac{1}{2}\left(f_{a 30}-f_{b 30}\right), d k_{10} \equiv \frac{1}{2}\left(k_{130}-k_{330}\right)\right\}
$$

- those of dimension- 4 operators:

$$
\left\{f_{a 40}, g_{40}\right\}
$$

- that of a dimension-6 operator:

$$
\left\{f_{a 60}\right\}
$$

- that of a dimension-7 operator:

$$
\left\{k_{270}\right\},
$$

- and finally, that of a dimension- 8 operators:

$$
\left\{f_{a 80}\right\} \text {. }
$$

Introducing $y=1-x$, the regular horizon $y \rightarrow 0_{+}$asymptotics of

$$
\left\{K_{1}, K_{2}, K_{3}, f_{a}, f_{b}, f_{c}, h, g\right\},
$$

take form:

$$
\begin{aligned}
K_{i} & =\sum_{n=0}^{\infty} k_{i h n} y^{2 n}, & & i=1,2,3, \\
f_{\alpha} & =a_{0} \sum_{n=0}^{\infty} f_{\alpha h n} y^{2 n}, & \alpha & =a, b, c, \\
h & =\sum_{n=0}^{\infty} h_{h n} y^{2 n}, & g & =g_{0} \sum_{n=0}^{\infty} g_{h n} y^{2 n} .
\end{aligned}
$$


Here, the expansion is characterized by 9 parameters:

$$
\left\{k_{1 h 0}, k_{2 h 0}, k_{3 h 0}, f_{a h 0}, f_{b h 0}, f_{c h 0}, f_{c h 1}, h_{h 0}, g_{h 0}\right\} \text {. }
$$

Equations of motion are invariant under the following symmetries:

SYM-A:

$$
h \rightarrow \lambda^{-2} h, \quad f_{a, b, c} \rightarrow \lambda f_{a, b, c},
$$

- SYM-B:

$$
P \rightarrow \lambda^{-1} P, \quad g \rightarrow \lambda^{2} g
$$

- SYM-C:

$$
h \rightarrow \lambda^{2} h, f_{a, b, c} \rightarrow f_{a, b, c}, K_{1,3} \rightarrow \lambda^{2} K_{1,3}, K_{2} \rightarrow K_{2}, g \rightarrow g, P \rightarrow \lambda P
$$

Above rescaling symmetries can be used to set:

$$
P=g_{0}=a_{0}=1
$$

It is important to present physical results in dimensionless form - from (A.20) we see that with (A.31)

$$
\Lambda=e^{-k_{s} / 2}
$$

\section{A.2 Holographic renormalization, thermodynamic quantities and horizon $D 3$ Maxwell charge}

Holographic renormalization of the theory (A.1) with unbroken U(1) symmetry was implemented in [14]. To study KSBH we need renormalization with $U(1) \supset \mathbb{Z}_{2}$. For quantities of interest, this is easily done with the following substitutions:

$$
K^{K T}=\frac{1}{2} K_{1}+\frac{1}{2} K_{3}, \quad \Omega_{1}^{K T}=3 \Omega_{1}, \quad \Omega_{2}^{K T}=\frac{\sqrt{6}}{2}\left(\Omega_{2}+\Omega_{3}\right)
$$

For relevant thermodynamic quantities we find:

- the temperature $T$ :

$$
\begin{aligned}
\frac{T}{\Lambda}= & \frac{e^{k_{s} / 2}}{16 \pi}\left(-\frac{1}{f_{a h 0}^{2} f_{b h 0}^{2} g_{h 0} h_{h 0}^{2}\left(f_{c h 0}+2 f_{c h 1}\right)}\left(\left(16 f_{a h 0}^{2} k_{2 h 0}^{2}\right.\right.\right. \\
& \left.+16 f_{b h 0}^{2} k_{2 h 0}^{2}-64 f_{b h 0}^{2} k_{2 h 0}+64 f_{b h 0}^{2}\right) g_{h 0}^{2}-16 f_{a h 0} f_{b h 0} h_{h 0} \\
& \left(3 f_{b h 0}-3 f_{a h 0}+4 f_{c h 0}\right)\left(4 f_{c h 0}-3 f_{b h 0}+3 f_{a h 0}\right) g_{h 0} \\
& \left.\left.+18 f_{a h 0} f_{b h 0} k_{1 h 0}^{2}-36 f_{a h 0} f_{b h 0} k_{1 h 0} k_{3 h 0}+18 f_{a h 0} f_{b h 0} k_{3 h 0}^{2}\right)\right)^{1 / 2}
\end{aligned}
$$

- the entropy density $s$ :

$$
16 \pi G_{5} \frac{s}{\Lambda^{3}}=4 \pi e^{3 k_{s} / 2} h_{h 0}^{1 / 2} f_{c h 0}^{1 / 2} f_{a h 0} f_{b h 0}
$$


- the energy density $\mathcal{E}$ :

$$
16 \pi G_{5} \frac{\mathcal{E}}{\Lambda^{4}}=e^{2 k_{s}}\left(3-12 f_{a 40}\right)
$$

- the free energy density $\mathcal{F}$ :

$$
\mathcal{F}=\mathcal{E}-s T
$$

A highly nontrivial check is a numerical verification of the first law of the thermodynamics. We find (for constant $\Lambda$ )

$$
\left|1-\frac{T d s}{d \mathcal{E}}\right|_{\text {numerically }}=\left|1-\frac{\frac{T}{\Lambda} d\left(s / \Lambda^{3}\right)}{d\left(\mathcal{E} / \Lambda^{4}\right)}\right|_{\text {numerically }} \sim 5 \times 10^{-6} \cdots 3 \times 10^{-5}
$$

From [13]

$$
16 \pi G_{5}=\frac{128 \pi^{4}}{81 P^{4}}
$$

From [21] the Maxwell charge at the black hole horizon is

$$
Q_{b}^{\mathrm{D} 3}=\frac{1}{54 \pi}\left(k_{1 h 0}\left(2-k_{2 h 0}\right)+k_{2 h 0} k_{3 h 0}\right)
$$

\section{A.3 Numerical procedure}

We are now ready to formulate our numerical procedure, and count the parameters of the solution:

- We integrate the differential equations along $x$-coordinate

$$
0 \leq x \leq 1
$$

with $x=0$ being the boundary and $x=1$ being the horizon.

- We use various scaling symmetries discussed above to set (A.31).

- Altogether we need to integrate 8 functions

$$
\left\{K_{1}, K_{2}, K_{3}, f_{a}, f_{b}, f_{c}, h, g\right\},
$$

for a given set of the remaining microscopic parameter $\left\{k_{s}\right\}$.

- The solution is then determined by 7 UV parameters (A.21)-(A.25), and 9 IR parameters (A.27):

$$
\begin{aligned}
\mathrm{UV}: & \left\{d f_{0}, d k_{10}, f_{a 40}, g_{40}, f_{a 60}, k_{270}, f_{a 80}\right\}, \\
\mathrm{IR}: & \left\{k_{1 h 0}, k_{2 h 0}, k_{3 h 0}, f_{a h 0}, f_{b h 0}, f_{c h 0}, f_{c h 1}, h_{h 0}, g_{h 0}\right\} .
\end{aligned}
$$

Overall we have 16 parameters, precisely what is necessary to determine (A.42) from the appropriate second order differential equations.

We follow numerical method introduced in [13]. In a nutshell, for a fixed microscopic parameter $\left\{k_{s}\right\}$, we choose a 'trial' set of parameters (A.43) and integrate (a double set of) 
the equations of motion for (A.42) from the UV $\left(x_{\text {initial }}=0.001\right)$ to $x=0.5$, and from the IR $\left(y_{\text {initial }}=0.001\right)$ to $y=0.5$. A solution (A.43) of the boundary value problem implies that the mismatch vector

$$
\begin{aligned}
\vec{v}_{\text {mismatch }} \equiv & \left(K_{1}^{b}-K_{1}^{h},\left(K_{1}^{b}+K_{1}^{h}\right)^{\prime}, K_{2}^{b}-K_{2}^{h},\left(K_{2}^{b}+K_{2}^{h}\right)^{\prime}, K_{3}^{b}-K_{3}^{h},\right. \\
& \left(K_{3}^{b}+K_{3}^{h}\right)^{\prime}, f_{a}^{b}-f_{a}^{h},\left(f_{a}^{b}+f_{a}^{h}\right)^{\prime}, f_{b}^{b}-f_{b}^{h},\left(f_{b}^{b}+f_{b}^{h}\right)^{\prime}, f_{c}^{b}-f_{c}^{h}, \\
& \left.\left(f_{c}^{b}+f_{c}^{h}\right)^{\prime}, h^{b}-h^{h},\left(h^{b}+h^{h}\right)^{\prime}, g^{b}-g^{h},\left(g^{b}+g^{h}\right)^{\prime}\right)_{x=y=0.5},
\end{aligned}
$$

with the superscripts ${ }^{b}$ and ${ }^{h}$ referring to the boundary (UV) and the horizon (IR) integrations, vanishes. At each iteration we adjust the set of parameters (A.43) along the direction of the steepest decent for $\left\|\vec{v}_{\text {mismatch }}\right\|$. In practice, for a valid numerical solution we were able to achieve

$$
\left\|\vec{v}_{\text {mismatch }}\right\| \sim 10^{-37} \cdots 10^{-32} .
$$

Open Access. This article is distributed under the terms of the Creative Commons Attribution License (CC-BY 4.0), which permits any use, distribution and reproduction in any medium, provided the original author(s) and source are credited.

\section{References}

[1] P. Candelas and X.C. de la Ossa, Comments on Conifolds, Nucl. Phys. B 342 (1990) 246 [INSPIRE].

[2] J.M. Maldacena, The large $N$ limit of superconformal field theories and supergravity, Int. J. Theor. Phys. 38 (1999) 1113 [hep-th/9711200] [INSPIRE].

[3] I.R. Klebanov and E. Witten, Superconformal field theory on three-branes at a Calabi-Yau singularity, Nucl. Phys. B 536 (1998) 199 [hep-th/9807080] [INSPIRE].

[4] I.R. Klebanov and M.J. Strassler, Supergravity and a confining gauge theory: Duality cascades and $\chi S B$ resolution of naked singularities, JHEP 08 (2000) 052 [hep-th/0007191] [INSPIRE].

[5] N. Seiberg, Electric-magnetic duality in supersymmetric nonAbelian gauge theories, Nucl. Phys. B 435 (1995) 129 [hep-th/9411149] [INSPIRE].

[6] S.B. Giddings, S. Kachru and J. Polchinski, Hierarchies from fluxes in string compactifications, Phys. Rev. D 66 (2002) 106006 [hep-th/0105097] [INSPIRE].

[7] S. Kachru, R. Kallosh, A.D. Linde and S.P. Trivedi, de Sitter vacua in string theory, Phys. Rev. D 68 (2003) 046005 [hep-th/0301240] [InSPIRE].

[8] E. Witten, Anti-de Sitter space, thermal phase transition and confinement in gauge theories, Adv. Theor. Math. Phys. 2 (1998) 505 [hep-th/9803131] [INSPIRE].

[9] A. Buchel, Finite temperature resolution of the Klebanov-Tseytlin singularity, Nucl. Phys. B 600 (2001) 219 [hep-th/0011146] [InSPIRE].

[10] I.R. Klebanov and A.A. Tseytlin, Gravity duals of supersymmetric $\mathrm{SU}(N) \times \mathrm{SU}(N+M)$ gauge theories, Nucl. Phys. B 578 (2000) 123 [hep-th/0002159] [INSPIRE]. 
[11] A. Buchel, C.P. Herzog, I.R. Klebanov, L.A. Pando Zayas and A.A. Tseytlin, Nonextremal gravity duals for fractional D-3 branes on the conifold, JHEP 04 (2001) 033 [hep-th/0102105] [INSPIRE].

[12] S.S. Gubser, C.P. Herzog, I.R. Klebanov and A.A. Tseytlin, Restoration of chiral symmetry: A supergravity perspective, JHEP 05 (2001) 028 [hep-th/0102172] [INSPIRE].

[13] O. Aharony, A. Buchel and P. Kerner, The black hole in the throat: Thermodynamics of strongly coupled cascading gauge theories, Phys. Rev. D 76 (2007) 086005 [arXiv:0706.1768] [INSPIRE].

[14] O. Aharony, A. Buchel and A. Yarom, Holographic renormalization of cascading gauge theories, Phys. Rev. D 72 (2005) 066003 [hep-th/0506002] [INSPIRE].

[15] A. Buchel, Hydrodynamics of the cascading plasma, Nucl. Phys. B 820 (2009) 385 [arXiv: 0903.3605] [INSPIRE].

[16] A. Buchel, Chiral symmetry breaking in cascading gauge theory plasma, Nucl. Phys. B 847 (2011) 297 [arXiv: 1012 .2404] [InSPIRE].

[17] I. Bena, M. Graña and N. Halmagyi, On the Existence of Meta-stable Vacua in Klebanov-Strassler, JHEP 09 (2010) 087 [arXiv:0912.3519] [INSPIRE].

[18] I. Bena, G. Giecold, M. Graña, N. Halmagyi and S. Massai, On Metastable Vacua and the Warped Deformed Conifold: Analytic Results, Class. Quant. Grav. 30 (2013) 015003 [arXiv: 1102.2403] [INSPIRE].

[19] I. Bena, G. Giecold, M. Graña, N. Halmagyi and S. Massai, The backreaction of anti-D3 branes on the Klebanov-Strassler geometry, JHEP 06 (2013) 060 [arXiv:1106.6165] [INSPIRE].

[20] I. Bena, M. Graña, S. Kuperstein and S. Massai, Anti-D3 Branes: Singular to the bitter end, Phys. Rev. D 87 (2013) 106010 [arXiv:1206.6369] [InSPIRE].

[21] I. Bena, A. Buchel and O.J.C. Dias, Horizons cannot save the Landscape, Phys. Rev. D 87 (2013) 063012 [arXiv:1212.5162] [INSPIRE].

[22] S.S. Gubser, Curvature singularities: The good, the bad and the naked, Adv. Theor. Math. Phys. 4 (2000) 679 [hep-th/0002160] [InSPIRE].

[23] A. Dymarsky, I.R. Klebanov and N. Seiberg, On the moduli space of the cascading $\mathrm{SU}(M+p) \times \mathrm{SU}(P)$ gauge theory, JHEP 01 (2006) 155 [hep-th/0511254] [INSPIRE].

[24] A. Buchel, A holographic perspective on Gubser-Mitra conjecture, Nucl. Phys. B 731 (2005) 109 [hep-th/0507275] [INSPIRE].

[25] M. Mia, F. Chen, K. Dasgupta, P. Franche and S. Vaidya, Non-Extremality, Chemical Potential and the Infrared limit of Large N Thermal QCD, Phys. Rev. D 86 (2012) 086002 [arXiv: 1202.5321] [INSPIRE].

[26] A. Buchel and C. Pagnutti, Exotic Hairy Black Holes, Nucl. Phys. B 824 (2010) 85 [arXiv: 0904.1716] [INSPIRE].

[27] P. Bosch, A. Buchel and L. Lehner, Unstable horizons and singularity development in holography, JHEP 07 (2017) 135 [arXiv: 1704.05454] [INSPIRE].

[28] A. Buchel, Singularity development and supersymmetry in holography, JHEP 08 (2017) 134 [arXiv: 1705.08560] [INSPIRE]. 